SPIE Proceedings, San Diego, CA, July 2000.

\title{
On Nonuniform Principal Component Filter Banks: Definitions, Existence and Optimality
}

\author{
Sony Akkarakaran and P.P.Vaidyanathan \\ Department of Electrical Engineering 136-93, \\ California Institute of Technology, \\ Pasadena, CA-91125, U.S.A. \\ Email: sony@systems.caltech.edu,ppvnath@sys.caltech.edu
}

\begin{abstract}
The optimality of principal component filter banks (PCFB's) for data compression has been observed in many works to varying extents. Recent work by the authors has made explicit the precise connection between the optimality of uniform orthonormal filter banks (FB's) and the principal component property: The PCFB is optimal whenever the minimization objective is a concave function of the subband variances of the FB. This gives a unified explanation of PCFB optimality for compression, denoising and progressive transmission. However not much is known for the case when the optimization is over a class of nonuniform FB's. In this paper we first define the notion of a PCFB for a class of nonuniform orthonormal FB's. We then show how it generalizes the uniform PCFB's by being optimal for a certain family of concave objectives. Lastly, we show that existence of nonuniform PCFB's could imply severe restrictions on the input power spectrum. For example, for the class of unconstrained orthonormal nonuniform FB's with any given set of decimators that are not all equal, there is no PCFB if the input spectrum is strictly monotone.
\end{abstract}

Keywords: Principal Component Filter-Banks, nonuniform filter banks, optimal filter banks, majorization, convex objectives

\section{INTRODUCTION}

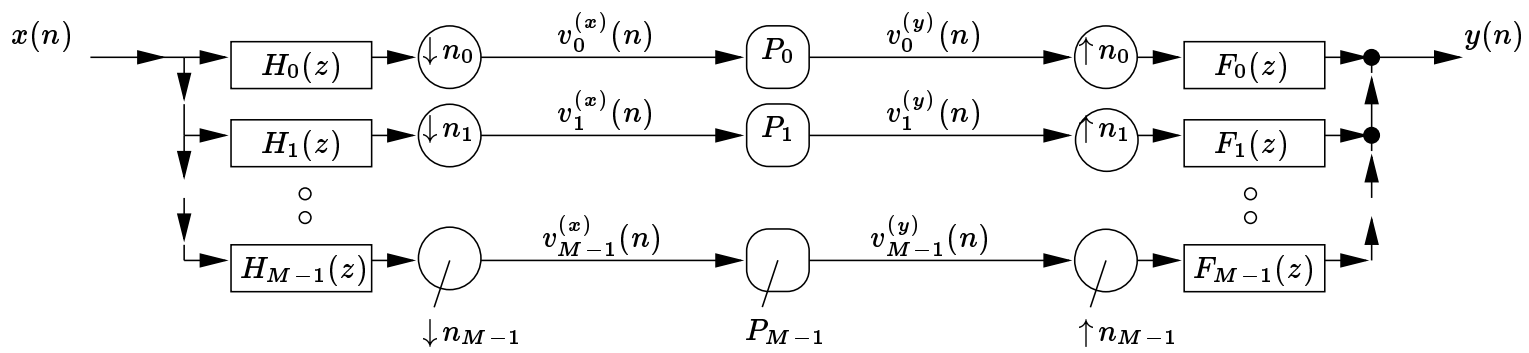

Fig. 1. General subband signal processing scheme using $M$ channel filter bank.

Figure 1 shows a general subband signal processing scheme using an $M$ channel filter bank (FB). We always assume the FB to be maximally decimated, i.e. the channel decimation rates $n_{i}$ satisfy

$$
\sum_{i=0}^{M-1} \frac{1}{n_{i}}=1 .
$$

The FB is uniform if all decimators $n_{i}$ are equal, i.e. (by (1)) $n_{i}=M$ for all $i$. The FB has the perfect reconstruction (PR) property if the input and output are identical in absence of subband processing, i.e. in Fig. $1, y(n) \equiv x(n)$ when all the $P_{i}$ are identity systems. We will only study orthonormal FB's, i.e. those having PR with $F_{i}\left(e^{j \omega}\right)=H_{i}^{*}\left(e^{j \omega}\right)$.

The subband processors $P_{i}$ are usually aimed at producing a certain desired signal $d(n)$ at the FB output. For example in data compression, the $P_{i}$ are quantizers and $d(n)=x(n)$. In noise suppression, $x(n)=s(n)+\mu(n)$ where

Work supported in parts by the National Science Foundation under Grant MIP 0703755, and by Microsoft Research, Redmond, WA. 
$\mu(n)$ is the additive noise, and $d(n)=s(n)$, the pure signal. Now let us be given a class of FB's, all having the same set of decimators $n_{i}$. The question arises as to which is the best FB in the class for a given application. In other words, given the processors $P_{i}$ and some well defined measure of the error signal $e(n)=y(n)-d(n)$ between the true and desired FB output, which FB in the class minimizes the error measure? The answer of course depends on the error measure and the given FB class.

To obtain a well defined error measure, it is common to employ statistical models of the input using stationary random processes. In many situations the error signal $e(n)$ is cyclostationary, and the error measure used is its average mean-square value. Now suppose the given FB class is uniform, i.e. all decimators have same value $M$. For such classes, it often happens that the above mentioned mean square value is a concave function of the subband variances. Recently it has been shown ${ }^{1,2}$ that in all such cases, a principal component filter bank (PCFB) ${ }^{10-12,1}$ for the given FB class and input spectrum will be optimal (if it exists). This leads to a unified explanation of PCFB optimality for several applications such as progressive transmission, compression, noise suppression, and as shown more recently, for optimal DMT (discrete multitone modulation) communications. ${ }^{13}$

The present work seeks to generalize this basic PCFB optimality result to classes of nonuniform FB's. In $^{3}$ a preliminary study of nonuniform PCFB's has been carried out by the authors. This paper elaborates on these results. We compare our definitions of nonuniform PCFB's with previously attempted definitions ${ }^{8,14}$ that are superficially similar to that of uniform PCFB's. We point out that these earlier works do not involve the central concept of majorization $^{1,7}$ which is crucial to uniform PCFB optimality. Thus the defined nonuniform PCFB's could not be shown to have any interesting optimality properties analogous to uniform ones. Using our definitions, we can show certain general optimality properties for concave objectives. The form of the objective is somewhat more restricted here as compared to that for uniform FB classes, however the results clearly show that our definitions are the more natural generalization of PCFB's to classes of nonuniform FB's. We will then prove an important result stating that for strictly monotone input power spectra, PCFB's do not exist for the class of unconstrained nonuniform FB's with any given set of decimators that are not all equal. In contrast, the class of unconstrained uniform $M$-channel orthonormal FB's always has a PCFB for any input spectrum. Thus PCFB existence for nonuniform FB classes is much more delicate than that for uniform ones.

\section{PROBLEM FORMULATION}

Consider first the case when the FB is uniform, i.e. $n_{i}=M$ for all $i$ in Fig. 1. Denote by $v_{i}^{(s)}(n)$ the $i$-th subband signal when the FB input is $s(n)$. We are given a class of (uniform $M$-channel) orthonormal FB's, and the power spectrum (psd) of the wide sense stationary (WSS) FB input $x(n)$. As explained earlier, we seek to find the best FB in the class for the given psd. Suppose the subband error signals $v_{i}^{(e)}(n)$ are jointly WSS, or equivalently the full band error $e(n)$ is wide sense cyclostationary with period $M(\operatorname{CWSS}(M))$. From FB orthonormality it can be shown that the chosen minimization objective, i.e. time averaged expected mean square value $\varepsilon$ of $e(n)$, has the form

$$
\varepsilon=\frac{1}{M} \sum_{i=0}^{M-1} E\left[\left|v_{i}^{(e)}(n)\right|^{2}\right] .
$$

Often the subband error variance is related to the subband signal variance $\sigma_{i}^{2} \triangleq E\left[\left|v_{i}^{(x)}(n)\right|^{2}\right]$, as

$$
E\left[\left|v_{i}^{(e)}(n)\right|^{2}\right]=h_{i}\left(\sigma_{i}^{2}\right)
$$

where $h_{i}$ is some function depending purely on the nature of the subband processing (i.e. on $P_{i}$ in Fig. 1), and independent of the choice of FB.* Thus in this case, the minimization objective $g$ is purely a function of the subband variance vector $\mathbf{v} \triangleq\left(\sigma_{0}^{2}, \sigma_{1}^{2}, \ldots, \sigma_{M-1}^{2}\right)$, i.e.

$$
g(\mathbf{v})=\frac{1}{M} \sum_{i=0}^{M-1} h_{i}\left(\sigma_{i}^{2}\right)
$$

Now if all the functions $h_{i}$ are concave on the interval $[0, \infty)$ then $g(\mathbf{v})$ is a concave function of the variance vector v. In this case, a principal component filter bank (PCFB) for the given class of FB's and given input psd will be optimal (if it exists). Uniform PCFB's and this central result on their optimality will be reviewed in the next section.

*The term 'variance' here should technically be replaced with 'energy' or expected mean square value; we will often make this convenient misuse of terminology. The distinction vanishes if the relevant signals are assumed to be zero mean. 
(a)
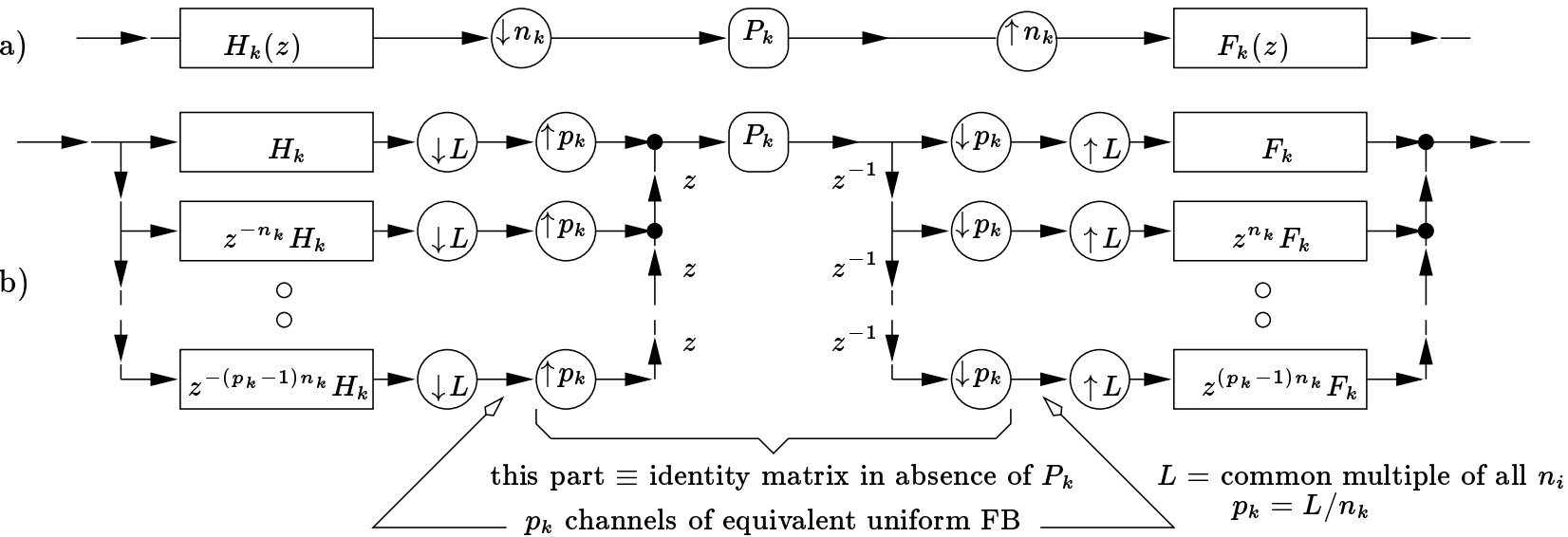

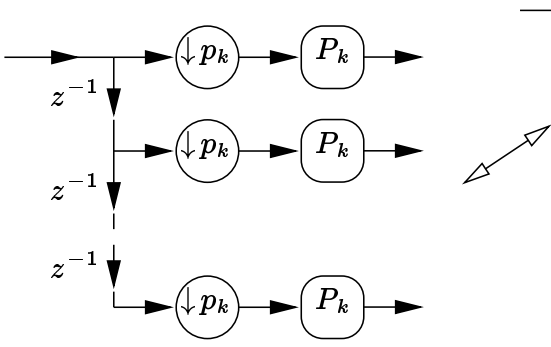

(c)
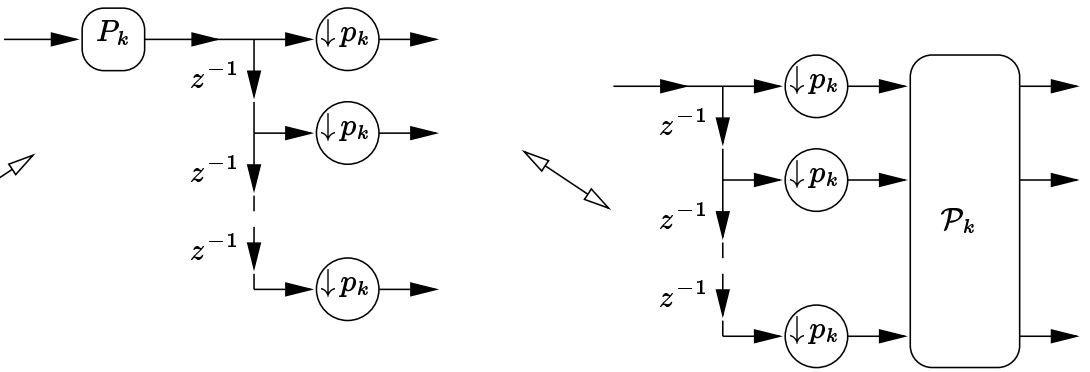

(d)

Fig. 2. Transforming nonuniform FB's to equivalent uniform ones.

(a) A single subband, (b) corresponding subbands of equivalent uniform FB, (c) moving processor $P_{k}$ from nonuniform to uniform subbands - special case, (d) general case.

Now consider the case when all FB's in the given class of orthonormal $M$-channel FB's are nonuniform with a fixed set of decimators $n_{i}$ (not necessarily all equal). In this section we will (a) generalize the form of the objective (4) to cover this case, and (b) illustrate the generalization using specific signal processing problems where the assumptions leading to (4) are satisfied for uniform FB classes.

The key step in dealing with nonuniform FB classes is the transformation ${ }^{6,4,9}$ from the nonuniform FB to a uniform $L$-channel FB where $L$ is any common multiple of the decimators $n_{i}$ (usually $L=\operatorname{lcm}\left\{n_{i}\right\}$ ). This transformation is shown in Fig. 2a,b. The $k$-th channel of the nonuniform FB, with decimator $n_{k}$, corresponds to $p_{k}=L / n_{k}$ channels of the uniform one. The filters in these $p_{k}$ channels are delayed versions of each other. Due to these dependencies between the filters of the equivalent uniform FB, it is not possible to redraw every $L$-channel uniform $\mathrm{FB}$ as a nonuniform FB with the given decimators $n_{i}$ (unless we allow the nonuniform FB to have filters that are periodically time varying ${ }^{4}$ with period $L$ ). Note from Fig. 2 that many properties such as maximal decimation, perfect reconstruction and orthonormality are shared in common by the nonuniform FB and its equivalent uniform FB (i.e. each has the property if and only if the other does).

The above transformation makes it easy to formulate the minimization objective for nonuniform FB's starting from the earlier formulation for uniform ones. Let $w_{i}^{(s)}(n)$ denote the signals in the equivalent uniform FB subbands when the FB input is $s(n)$. The earlier notation $v_{i}^{(s)}(n)$ is used for the nonuniform FB subbands. Both (2) and (3) can be generalized to nonuniform FB's; the issue to be resolved is whether the subband signals in these equations should now refer to the uniform subbands $w_{i}^{(e)}(n)$ or to the nonuniform ones $v_{i}^{(e)}(n)$. Evidently (2) generalizes as $\varepsilon=\sum_{i=0}^{L-1} E\left[\left|w_{i}^{(e)}(n)\right|^{2}\right]$, under the assumption that the signals $w_{i}^{(e)}(n)$ are jointly WSS. On the other hand, (3) reflects the action of processor $P_{i}$ on its WSS input (a subband signal), and hence generalizes as it is, under the assumption that the signal $v_{i}^{(x)}(n)$ is WSS with variance $\sigma_{i}^{2}$. Finally, examine the signals $w_{i}^{(e)}(n)$ for the group of $p_{k}$ uniform FB subbands in Fig. 2b. By linearity of the systems in Fig. 2, these signals are obtained by passing 
the signal $v_{k}^{(e)}(n)$ of the corresponding nonuniform subband through the $p_{k}$ channel delay-chain. Thus if $v_{k}^{(e)}(n)$ is WSS, all these $w_{i}^{(e)}(n)$ have the same variance equal to that of $v_{k}^{(e)}(n)$. Combining these observations yields the minimization objective for nonuniform FB classes:

$$
g(\mathbf{v})=\sum_{i=0}^{M-1} \frac{p_{i}}{L} h_{i}\left(\sigma_{i}^{2}\right)=\sum_{i=0}^{M-1} \frac{1}{n_{i}} h_{i}\left(\sigma_{i}^{2}\right)
$$

This is a very natural generalization of (4), obtained by appropriate generalization of the choice of stationarity assumptions.

Notice that (5) is exactly the objective that would result for the equivalent uniform FB optimization if the processor $P_{k}$ in Fig. 2b were replaced by $p_{k}$ identical copies of itself, one in each of the corresponding uniform FB subbands as demonstrated in Fig. 2c. For a general processor $P_{k}$, this operation would be changing the system if the system is to be preserved a $p_{k}$-input $p_{k}$-output processor is needed in the uniform $\mathrm{FB}$ subbands as shown in Fig. 2d. However the two systems are identical from the point of view of the minimization objective. We now mention specific signal processing schemes satisfying the assumptions leading to (5), the corresponding processors $P_{i}$ and 'error functions' $h_{i}$. For all these $P_{k}$ the two systems in Fig. $2 \mathrm{c}$ in fact happen to be equivalent. Also the $h_{i}$ turn out to be concave on $[0, \infty)$ so that in the special case of uniform FB classes $\left(n_{i}=M\right.$ for all $\left.i\right)$, PCFB's are optimal (Section 3.1). The later sections seek to generalize this to nonuniform FB classes.

Compression: Here the processor $P_{i}$ is a quantizer, modelled as an additive noise source with variance proportional to the variance $\sigma_{i}^{2}$ of the (WSS) quantizer input. The proportionality constant $f_{i}\left(b_{i}\right)$ depends on the number of bits $b_{i}$ alloted to the quantizer, and is assumed to be independent of choice of FB. Thus $h_{i}(x)=f_{i}\left(b_{i}\right) x$ in $(5)$. For uniform FB's, we need the assumption that the noises from different quantizers are all jointly WSS. For nonuniform FB's, we require the corresponding noises generated in the subbands of the equivalent uniform FB to be jointly WSS. The stronger assumption of WSS and uncorrelated quantizer noises implies both these.

The special case where the quantizers are assumed to satisfy the high bit-rate assumption is worth mention: Here $f_{i}\left(b_{i}\right)=c_{i} 2^{-2 b_{i}}$ where the constant depends on the $i$-th subband probability density function (pdf), and hence on the choice of FB. This is not allowed under our formulation of (5) (the functions $h_{i}$ are assumed to be independent of choice of FB); however we circumvent the problem by assuming a Gaussian input. This forces all subband pdf's to be Gaussian independent of the $\mathrm{FB}$, so that $c_{i}=c$ for all $i$, where $c$ is a constant independent of the FB. In this case, under optimal allocation of the bits $b_{i}$ (subject to a total bitrate constraint $\sum_{i=0}^{M-1} b_{i}=B$ ), it can be shown (using the arithmetic mean - geometric mean inequality) that minimizing (5) is equivalent to minimizing the weighted geometric mean $G M=\prod_{i=0}^{M-1}\left(\sigma_{i}^{2}\right)^{\left(1 / n_{i}\right)}$ of the subband variances $\sigma_{i}^{2}$ (with the decimators $n_{i}$ as weights). Equivalently, we can minimize $\log (G M)$, which again has the form of $(5)$ with $h_{i}(x)=\log (x)$ for all $i$.

Noise reduction: Here the FB input is $x(n)=s(n)+\mu(n)$ where $s(n)$ is the pure input and $\mu(n)$ is white noise uncorrelated to $s(n)$. Each processor $P_{i}$ is a multiplier $k_{i}$ that is either constant or adapted to its input statistics as (a) a Wiener filter: $k_{i}=\frac{\sigma_{i}^{2}}{\sigma_{i}^{2}+\eta^{2}}$, or (b) a subband hard threshold operator: $k_{i}=0$ if $\sigma_{i}^{2}<\eta^{2}$ and $k_{i}=1$ otherwise. Here $\sigma_{i}^{2}$ is the variance of the pure signal component $v_{i}^{(s)}(n)$ in the $i$-th subband, and $\eta^{2}$ is the noise variance. Equation (5) applies in all cases, with

$$
h_{i}(x)=\left\{\begin{array}{cl}
\left|1-k_{i}\right|^{2} x+\left|k_{i}\right|^{2} \eta^{2} & \text { for constant subband multiplier } k_{i} \\
\frac{x \eta^{2}}{x+\eta^{2}} & \text { for zeroth order Wiener filter } k_{i} \\
\min \left(x, \eta^{2}\right) & \text { for hard threshold } k_{i}
\end{array}\right.
$$

\section{NONUNIFORM PCFB'S: DEFINITIONS AND OPTIMALITY}

\subsection{Review of uniform PCFB's}

Definition: Majorization. Given two (equal length) vectors $\mathbf{a}=\left(a_{0}, a_{1}, \ldots, a_{M-1}\right)$ and $\mathbf{b}=\left(b_{0}, b_{1}, \ldots, b_{M-1}\right)$, we say that $\mathbf{a}$ majorizes $\mathbf{b}$ if after rearranging the entries of the vectors in decreasing order we have

$$
\sum_{i=0}^{P} a_{i} \geq \sum_{i=0}^{P} b_{i} \quad \text { for } \quad 0 \leq P \leq M-1, \quad \text { with equality holding for } P=M-1
$$


Note that if $\mathbf{a}$ majorizes $\mathbf{b}$, any permutation of $\mathbf{a}$ majorizes any permutation of $\mathbf{b}$.

Definition: Uniform PCFB's. Let $\mathcal{C}$ be a class of uniform orthonormal $M$-channel FB's. A FB in $\mathcal{C}$ is said to be a PCFB for $\mathcal{C}$ for the given input power spectrum if its subband variance vector $\left(\hat{\sigma}_{0}^{2}, \hat{\sigma}_{1}^{2}, \ldots, \hat{\sigma}_{M-1}^{2}\right)$ majorizes the subband variance vector $\left(\sigma_{0}^{2}, \sigma_{1}^{2}, \ldots, \sigma_{M-1}^{2}\right)$ of every $\mathrm{FB}$ in $\mathcal{C}$.

The PCFB and its existence depends both on $\mathcal{C}$ and on the input psd. The requirement of majorization in the PCFB definition in particular demands that the average (or the sum) of the subband variances be FB-independent. This follows automatically from FB orthonormality: The average equals the variance of the WSS input to the FB.

PCFB optimality. PCFB's are optimum orthonormal FB's if the minimization objective is a concave function of the subband variance vector. As noted earlier, this basic result leads to PCFB optimality for many FB based signal processing schemes, motivating generalization of the PCFB concept to nonuniform FB's. Detailed proofs of (uniform) PCFB optimality ${ }^{1,2}$ are based on the geometric meaning of majorization. For the present purpose of generalization to nonuniform FB's, it suffices to note that uniform PCFB optimality is solely dependent on the following facts:

1. The objective function has form (4) where all $h_{i}$ are $F B$-independent functions concave on the interval $[0, \infty)$.

2. The PCFB subband variance vector majorizes the subband variance vectors of all FB's in the given class $\mathcal{C}$. (Definition of PCFB's)

3. The entries of the variance vector are allowed to be inserted in the objective (4) in any order. In other words, if $\mathbf{v}$ is a variance vector realizable by the given class $\mathcal{C}$, so is any permutation of $\mathbf{v}$. This is true because permuting $\mathbf{v}$ simply corresponds to permuting the subbands, or to redistributing the processors among them. The subbands have no intrinsic ordering - their numbering purely denotes their association with the subband processor. Clearly all permutations of the PCFB are PCFB's. In general they do not all have the same value for the objective (4); in fact a bad permutation could even give the worst possible performance. However the best one of these (finitely many) permutations will be optimal for the entire class $\mathcal{C}$.

\subsection{Nonuniform PCFB's: Definition}

We seek to generalize the definition of PCFB's to a general class $\mathcal{N}$ of nonuniform $M$-channel orthonormal FB's with a fixed set of decimators $n_{i}$. It is desirable that the generalization reduce to the usual definition for uniform FB's if all the $n_{i}$ are equal, and that the defined PCFB's be optimal for some reasonable class of objectives of the form (5). From the discussion of uniform PCFB optimality, a natural approach to generalization is to define some suitable variance vector for all FB's in the class, and define the PCFB to be the one whose variance vector majorizes all the variance vectors of all FB's in the class $\mathcal{N}$. This PCFB would then be optimal for all objectives of the form $\sum_{i} f_{i}\left(\alpha_{i}\right)$ where $\alpha_{i}$ are the entries of the variance vector, provided conditions analogous to facts 1,3 mentioned in the earlier section hold. The majorization requirement demands that $\sum_{i} \alpha_{i}$ be FB-independent; this should follow automatically from FB orthonormality just as it happens for uniform FB's. Otherwise it would impose too severe a constraint on the FB class $\mathcal{N}$, and PCFB's would hardly ever exist.

Let $\sigma_{i}^{2}$ be the variance of the $i$-th subband (with decimator $n_{i}$ ). Now FB orthonormality implies that $\sum_{i} \frac{\sigma_{i}^{2}}{n_{i}}$ is FB-independent, but in general $\sum_{i} \sigma_{i}^{2}$ is not (unless all the $n_{i}$ are equal). Thus, the usual definition of subband variance vector (as one with entries $\sigma_{i}^{2}$ ) is not suitable (unless the FB's are uniform). In fact the above considerations leave us only two reasonable definitions of the variance vector:

- The normalized subband variance vector defined as $\left(\frac{\sigma_{0}^{2}}{n_{0}}, \frac{\sigma_{1}^{2}}{n_{1}}, \ldots, \frac{\sigma_{M-1}^{2}}{n_{M-1}}\right)$.

- The equivalent uniform subband variance vector, defined as the (usual) subband variance vector of the equivalent uniform $L$-channel FB (for any fixed $L$ that is a multiple of all the $n_{i}$ ). From the construction of the equivalent uniform FB's (Fig. 2), with $p_{i}=L / n_{i}$, this vector has the form

$$
(\underbrace{\sigma_{0}^{2}, \sigma_{0}^{2}, \ldots, \sigma_{0}^{2}}_{p_{0} \text { elements }}, \quad \underbrace{\sigma_{1}^{2}, \sigma_{1}^{2}, \ldots, \sigma_{1}^{2}}_{p_{1} \text { elements }}, \quad \ldots \ldots, \quad \underbrace{\sigma_{M-1}^{2}, \sigma_{M-1}^{2}, \ldots, \sigma_{M-1}^{2}}_{p_{M-1} \text { elements }})^{T}
$$


Definition: Nonuniform PCFB's. Let $\mathcal{N}$ be a class of orthonormal $M$-channel FB's having a fixed set of decimation rates $n_{i}$, not necessarily equal. $\mathrm{A} F \mathrm{FB}$ in $\mathcal{N}$ is said to be a PCFB for $\mathcal{N}$ for the given input power spectrum if its suitably defined subband variance vector majorizes the subband variance vector of every FB in $\mathcal{C}$. The variance vector in this definition could be either the normalized or the equivalent uniform subband variance vector (defined earlier). Thus we have two definitions of nonuniform PCFB's, one based on each of these variance vectors.

\section{Remarks on the nonuniform PCFB definitions.}

1. Reduction to uniform PCFB's. For the special case of uniform FB's, where $n_{i}=M$ for all $i$, both the above definitions reduce to the usual one by choosing $L=M$ for the equivalent uniform variance vector and omitting the constant scale $M$ for the normalized variances.

2. Ambiguity in $L$ does not matter. Let $L_{1}=\operatorname{lcm}\left\{n_{i}\right\}$ and $L_{2}=k L_{1}$ for any positive integer $k$. For two FB's in the given class, let $\mathbf{v}_{1}^{*}$ and $\mathbf{v}_{1}$ be the variance vectors of their equivalent $L_{1}$ channel uniform FB's, and let $\mathbf{v}_{2}^{*}$ and $\mathbf{v}_{2}$ be the respective variance vectors for the equivalent $L_{2}$ channel uniform FB's. From (8), if the entries of $\mathbf{v}_{1}$ in descending order are $\left(a_{0}, a_{1}, \ldots, a_{L_{1}-1}\right)$ then the entries of $\mathbf{v}_{2}$ in descending order are obtained by arranging the $a_{i}$ in the same order and repeating each $a_{i} k$ times. Thus by definition (7) of majorization, $\mathbf{v}_{1}^{*}$ majorizes $\mathbf{v}_{1}$ if and only if $\mathbf{v}_{2}^{*}$ majorizes $\mathbf{v}_{2}$. Hence, the definition of PCFB's using the equivalent $L$ channel uniform subband variance vector is independent of which common multiple of the $n_{i}$ we fix $L$ to be.

3. The two PCFB definitions are distinct. It may seem that extending the above argument will also prove that using normalized variance vectors is equivalent to using the equivalent uniform ones. However this is not true. The reason is that the definition of majorization (7) demands arranging the entries of the vectors in decreasing order. This order could be different for the $\sigma_{i}^{2}$ (i.e. for the entries of (8)) and for the $\frac{\sigma_{i}^{2}}{n_{i}}$. The distinctness of the two definitions is shown $i^{3}$ by an example of a specific class $\mathcal{N}$ with exactly two FB's with decimators $2,4,4$, for a carefully chosen family of input spectra. Each FB is the unique PCFB by one definition but not by the other. In fact in this example the input psd can be chosen so that the PCFB defined by the equivalent uniform variances is a PCFB not just for the two element class $\mathcal{N}$, but for any class of FB's with decimators 2, 4, 4 to which it belongs. In other words it is a PCFB for the class of unconstrained orthonormal FB's with 2, 4,4 as decimators. ${ }^{\dagger}$ Even then, the other FB in $\mathcal{N}$ is the unique PCFB for $\mathcal{N}$ by the alternative definition.

\subsection{Nonuniform PCFB's: Optimality}

For uniform FB's, there is no intrinsic ordering of the subbands. Permuting the subbands, or redistributing the processors $P_{i}$ among the subbands, was simply represented by evaluating the same objective function $g(\mathbf{v})$ of $(4)$ for a different argument $\mathbf{v}$, i.e. a permutation of the original subband variance vector. On the other hand, nonuniform FB subbands are indexed by their decimation rates $n_{i}$. If we interchange the processors for two subbands with different decimators, the new performance measure must be computed by interchanging not just the corresponding variances but also the corresponding decimators in (5). This changes the functional form of the objective, since the objective in (5) is viewed as a function of the variances $\sigma_{i}^{2}$ with the $n_{i}$ as parameters. Free redistribution of processors among subbands without changing the functional form of the objective is possible only among groups of subbands with equal decimators. Thus, it is necessary to distinguish two different nonuniform FB optimization problems:

1. Finding the best FB for a fixed ordering of the subbands (i.e. of the $n_{i}$ ). We refer to this problem as $O P 1$.

2. Optimizing both the FB (i.e. its subband variances) and the decisions as to which subband should have which processor $P_{i}$, i.e the choice of ordering of the subbands (or the $n_{i}$ ). We refer to this problem as $O P 2$.

The problem $O P 1$ does not exist for uniform FB's since there is no intrinsic ordering of the subbands. If $O P 1$ is solvable for all the (finitely many) possible orderings of the subbands, then clearly $O P 2$ is solved by picking from these solutions the one with the best value of the performance measure. Note that $O P 2$ is usually of much more interest than $O P 1$, since fixing apriori which channel decimator should be associated with which processor $P_{i}$ is quite restrictive. However $O P 1$ is easier to analyze, due to the fixed functional form of its objective. A summary of all the

\footnotetext{
${ }^{\dagger}$ This is done by setting $p=b$ in Fig. $3 a$ of $^{3}$ and using Theorem 3 in that reference.
} 
optimality results for nonuniform PCFB's (to be derived next) can be found at the end of the section, and optimality there refers only to the (more interesting) problem $O P 2$. The derivations involve study of both problems.

Optimality of PCFB defined using equivalent uniform variance vector: We generically denote the entries of this variance vector by $\alpha_{i}$. The objective (5) has the form $\sum_{i} f_{i}\left(\alpha_{i}\right)$ with the $f_{i}$ chosen as follows: All the $p_{k}=L / n_{k}$ equivalent uniform FB subbands derived from the $k$-th nonuniform FB subband (with decimator $n_{k}$ ) have the corresponding $f_{i}$ equal to $h_{k}$ of (5) (upto constant scale $L$ ). Thus this PCFB is a possible candidate for solving $O P 1$ when all $h_{k}$ (and hence all $f_{i}$ ) are FB-independent and concave. However for optimality, we must be allowed to insert the $\alpha_{i}$ into the $f_{i}$ in any order in forming the sum $\sum_{i} f_{i}\left(\alpha_{i}\right)$ (condition 3 in Section 3.1). In actual fact we are only allowed certain permutations of the $\alpha_{i}$, corresponding to groups of nonuniform subbands with equal decimator value. Thus the PCFB solves $O P 1$ if the best ordering of the $\alpha_{i}$ happens to be one that is actually allowed.

Recall that solving $O P 1$ for all permutations of the $n_{k}$ solves $O P 2$. For a given permutation of the $n_{k}$, suppose the PCFB does not actually solve $O P 1$, i.e. the best ordering of the $\alpha_{i}$ above is not allowed. Then this ordering of the $\alpha_{i}$ gives an unattainable upper bound on the performance for the problem $O P 1$. Sometimes we may be able to solve $O P 2$ using just these bounds (instead of the true optima). Indeed if a known solution to $O P 1$ for one permutation of the $n_{k}$ outperforms all these bounds computed for all the other permutations, then it also solves $O P 2$.

In the noteworthy special case when $h_{k}=h$ for all $i$, i.e. all $h_{k}$ and hence all $f_{i}$ are identical, we see that all orderings of the $\alpha_{i}$ give the same performance measure $\sum_{i} f_{i}\left(\alpha_{i}\right)$. Thus in this case the PCFB will solve OP1 if the function $h$ is FB-independent and concave. This is true for all permutations of the $n_{k}$, and the corresponding optimal performance measures are identical too. Thus the PCFB also solves $O P 2$. In fact the distinction between $O P 1$ and $O P 2$ vanishes in this case. Identical $h_{k}$ usually results from similar or identical subband processors $P_{k}$.

Optimality of PCFB defined using normalized variance vector: We generically denote the entries of this variance vector by $\beta_{i}=\sigma_{i}^{2} / n_{i}$. The objective (5) has the form $\sum_{i} \hat{f}_{i}\left(\beta_{i}\right)$ where $\hat{f}_{i}(x)=\left(1 / n_{i}\right) h_{i}\left(n_{i} x\right)$. Thus, this PCFB is a candidate for solving $O P 1$ when the $h_{i}$ (and hence the $\hat{f}_{i}$ ) are FB-independent and concave. However, again optimality is assured only if the $\beta_{i}$ can be inserted in the sum $\sum_{i} \hat{f}_{i}\left(\beta_{i}\right)$ in any order. In actual fact we are only permitted to permute the $\beta_{i}$ within groups of channels having the same decimator $n_{i}$. Thus, as with the earlier definition, these PCFB's solve $O P 1$ if the best ordering of the $\beta_{i}$ turns out to be one that is permitted. The special case where all $\hat{f}_{i}$ are identical (which would make all orderings of the $\beta_{i}$ equally good) is not of much interest here: It does not commonly happen since $\hat{f}_{i}(x)=\left(1 / n_{i}\right) h_{i}\left(n_{i} x\right)$ which depends on $n_{i}$.

However, another special case is of interest, namely that where $h_{i}(x)=k_{i} x$ for some constants $k_{i}$ (for all $i$ ). Here $\hat{f}_{i}(x)=k_{i} x$ too, which has the speciality of being (concave and) independent of the decimators $n_{i}$. In general, to compute the performance measure after permuting two nonuniform subbands required not just permuting of the corresponding $\beta_{i}$, but also corresponding modifications of the $\hat{f}_{i}$ since they depended on the $n_{i}$. (This changed the functional form of the objective, and gave rise to the distinction between problems $O P 1$ and $O P 2$.) However if $\hat{f}_{i}$ are independent of the $n_{i}$, permuting the nonuniform subbands is fully equivalent to permuting the $\beta_{i}$. Thus, the nonuniform FB defined using the variances $\beta_{i}$ will solve $O P 2$ (for a suitable permutation of its subbands, i.e. ordering of the $\beta_{i}$ ).

\section{Nonuniform PCFB optimality: Summary.}

- Nonuniform PCFB's defined using the equivalent uniform subband variance vector are optimal for objectives of the form (5) when $h_{i}=h$ for all $i$, where $h$ is a FB-independent concave function. Such objectives occur with similar processing in the subbands, e.g. in the high bitrate coding problem with optimal bit allocation $(h(x)=\log (x))$ or in the noise suppression problem using either zeroth order Wiener filters $\left(h(x)=\frac{x \eta^{2}}{x+\eta^{2}}\right)$ or hard thresholds $\left(h(x)=\min \left(x, \eta^{2}\right)\right)$ in all subbands (see Section 2).

- Nonuniform PCFB's defined using the normalized variance vector are optimal for objectives of the form (5) where for all $i, h_{i}(x)=k_{i} x$ for constant $k_{i}$. Such objectives occur in the coding problem with fixed bit allocation when the high bitrate assumption is not necessarily used $\left(k_{i}=f_{i}\left(b_{i}\right)\right.$, see Section 2).

- For general objectives of the form (5) where $h_{i}$ are FB-independent and concave, it may be possible to show optimality of either of the PCFB's in a specific case, using the actual specifications of the $h_{i}$ and numerical 
values of the PCFB subband variances. Finite procedures to do this follow from the earlier discussion. However in general the procedure will be unsuccessful, and PCFB optimality is not assured.

\subsection{Previous attempts at defining nonuniform PCFB's}

In the authors' knowledge, the works ${ }^{8,14}$ are the only earlier attempts to define nonuniform PCFB's. These can be described as follows: Let $\mathcal{N}$ be the given class of $M$-channel orthonormal FB's, all having the same set of decimators $n_{i}$. First an ordering of the subbands of all FB's (i.e. of the $n_{i}$ ) is decided upon. Then with $\sigma_{i}^{2}$ as the variance of the $i$-th subband (with decimator $n_{i}$ ), the PCFB is defined as one whose variances $\hat{\sigma}_{i}^{2}$ satisfy

$$
\sum_{i=0}^{P} \frac{\hat{\sigma}_{i}^{2}}{n_{i}} \geq \sum_{i=0}^{P} \frac{\sigma_{i}^{2}}{n_{i}} \quad \text { for } P=0,1, \ldots, M-1, \quad \text { with (automatic) equality for } P=M-1
$$

While this equation is superficially similar to (7) that defines majorization, there is a very important difference: The $a_{i}$ and $b_{i}$ of (7) are arranged in decreasing order, whereas the analogous quantities $\frac{\hat{\sigma}_{i}^{2}}{n_{i}}$ and $\frac{\sigma_{i}^{2}}{n_{i}}$ in (9) are arranged according to the predefined ordering of the $n_{i}$. $\mathrm{In}^{14}$ the PCFB is defined only for classes of dyadic (or 'wavelet style') tree-structured FB's, and the $n_{i}$ are chosen in decreasing order. A construction procedure is outlined for the PCFB thus defined, when the FB class $\mathcal{N}$ is the unconstrained one. $\mathrm{In}^{8}$ the PCFB is defined with respect to the ordering of the $n_{i}$, i.e. there are several PCFB's defined as above, one for each ordering of the $n_{i}$.

Consider the case when the minimization objective is as in (5) with $h_{i}(x)=k_{i} x$ for constant $k_{i}$ (for all $i$ ). Consider the FB optimization problem OP1 defined in Section 3.3, where the $n_{i}$ are ordered apriori and the association between the $n_{i}$ and $k_{i}$ is thus forcibly fixed. Let $j_{i}$ be the permutation such that $k_{j_{0}} \leq k_{j_{1}} \leq \ldots k_{j_{M-1}}$. Then elementary algebra shows that the PCFB defined in $^{8}$ as above, for the permutation $j_{i}$ of the decimators $n_{i}$, is a solution for $O P 1$.

Indeed it suffices to prove this assuming $j_{i}=i$. This amounts to showing that $\sum_{i} k_{i} \hat{\sigma}_{i}^{2} / n_{i} \leq \sum_{i} k_{i} \sigma_{i}^{2} / n_{i}$ given (9) and $k_{i}<k_{i+1}$. The proof follows from the fact that

$$
\sum_{i=0}^{M-1} k_{i} \frac{\sigma_{i}^{2}}{n_{i}}=\sum_{i=0}^{M-2}\left(k_{i}-k_{i+1}\right) \sum_{l=0}^{i} \frac{\sigma_{l}^{2}}{n_{l}}+k_{M-1} \sum_{i=0}^{M-1} \frac{\sigma_{i}^{2}}{n_{i}} .
$$

In summary, the earlier definitions result in several PCFB's, one for each permutation of the decimators $n_{i}$, and in optimality for the problem $O P 1$ for linear objectives (i.e. $h_{i}(x)=k_{i} x$ in (5)). However, in general optimality for the more interesting problem $O P 2$ cannot be claimed unless all the PCFB's (for all permutations of $n_{i}$ ) exist (in which case the best one of these would solve $O P 2$ ). Apart from the very special result in ${ }^{14}$ (specific to unconstrained dyadic tree-structured FB classes and using a particular ordering of subbands), there are no general existence results known for these PCFB's. Further, since the above PCFB definition does not use the concept of majorization, there are no optimality results for more general concave functions $h_{i}$ besides $h_{i}(x)=k_{i} x$. These observations suggest that our definitions of Section 3.2 are more natural generalizations of the PCFB concept to nonuniform FB classes.

\section{EXISTENCE OF NONUNIFORM PCFB'S}

For the case of uniform FB's, PCFB's are known to exist for all input power spectra for only three special FB classes: Any class of two channel FB's, the class of $M$-channel orthogonal transform coders, and the class $\mathcal{C}^{u}$ of unconstrained $M$-channel orthonormal FB's (where there are no constraints on the filters besides those imposed by orthonormality, e.g. ideal brickwall filters are allowed). For the class $\mathcal{C}^{u}$, the PCFB is the one satisfying the two properties called spectral majorization and total decorrelation. ${ }^{12}$ For classes of DFT and cosine-modulated FB's, PCFB's do not exist for large families of input spectra. ${ }^{2}$

Much less is known about existence of nonuniform PCFB's. For nonuniform PCFB's defined using normalized variances, no general existence result is currently known. Thus, in this section nonuniform PCFB's always mean those defined using equivalent uniform ones. Some existence results are known for these. ${ }^{3}$ Let $\mathcal{N}$ be any class of nonuniform FB's with a given set of decimators $n_{i}$, and let $\mathcal{E}$ be the corresponding class of equivalent uniform FB's derived from FB's in $\mathcal{N}$. Then by definition, PCFB's for $\mathcal{N}$ and $\mathcal{E}$ are equivalent. Now if the PCFB for some FB class $\mathcal{C} \supset \mathcal{E}$ lies within $\mathcal{E}$, then it is also a PCFB for $\mathcal{E}$. For example we could choose $\mathcal{C}=\mathcal{C}^{u}$, the unconstrained class, whose PCFB has been well studied. This gives rise to the result of ${ }^{3}$ stating that any FB in $\mathcal{N}$ having white and totally decorrelated subbands is a PCFB for $\mathcal{N}$. The paper ${ }^{3}$ also gives examples of such PCFB's. 
This section is devoted to a discussion and proof of an important nonexistence result for nonuniform PCFB's. The result is mentioned without proof in $^{3}$ and is as follows:

Theorem 1: Let $\mathcal{N}^{u}$ be the class of unconstrained nonuniform FB's with a given set of decimators $n_{i}$ not all equal. Let the input spectrum $S\left(e^{j \omega}\right)$ be strictly monotonic (increasing or decreasing) for $a \leq \omega<a+2 \pi$ for some real $a$. Then there is no PCFB for the class $\mathcal{N}^{u}$.

\subsection{Discussion on Theorem 1}

Existence of nonuniform PCFB's is very delicate. As shown in Fig. 3, a slight perturbation of a monotone input spectrum for which a PCFB for $\mathcal{N}^{u}$ exists (eg, the flat or white spectrum) can change it to a spectrum that is strictly monotone (on an interval of form $[a, a+2 \pi)$ ), destroying existence of the PCFB. In contrast, for the uniform unconstrained FB class $\mathcal{C}^{u}$, PCFB's exist for all input spectra, and moreover usually several perturbations are possible on the spectrum without even changing the PCFB. Of course, most objective functions defined on the FB class have their values for each FB perturbed only slightly by small perturbations of the input spectrum. Thus, consider the FB of Fig. 3. Being a PCFB for spectrum $A$, it optimizes many concave objectives as explained in Section 3.3. For the perturbed spectrum $B$, the FB is no longer a PCFB, and so it will not optimize all these objectives. However it will be quite close to optimal if the perturbation is small enough.

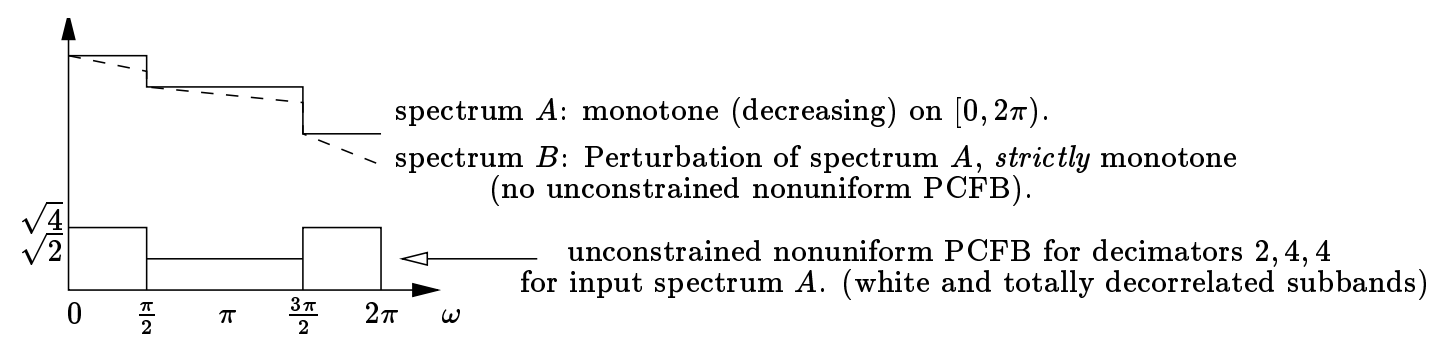

Fig. 3. Delicateness of existence of nonuniform PCFB for unconstrained class.

Total decorrelation and spectral majorization do not imply nonuniform PCFB's. Total decorrelation of subbands is evidently defined for nonuniform FB's too, and is achieved for example if the analysis filters have nonoverlapping supports. The notion of spectral majorization ${ }^{12}$ can also be defined for nonuniform FB's. (It is the condition when their subband spectra do not 'cross each other', just as for uniform FB's.) Thus, as shown in Fig. 4, if the input spectrum is monotone on $[a, a+2 \pi)$ then any contiguous-stacked brickwall FB with $a$ as a filter band-edge has subbands satisfying both these conditions. However the FB is not a PCFB for $\mathcal{N}^{u}$ if the spectrum is strictly monotone (since there is no PCFB in this case). Thus, unlike the situation for the class $\mathcal{C}^{u}$, FB's with subbands satisfying spectral majorization and total decorrelation need not be PCFB's for $\mathcal{N}^{u}$.
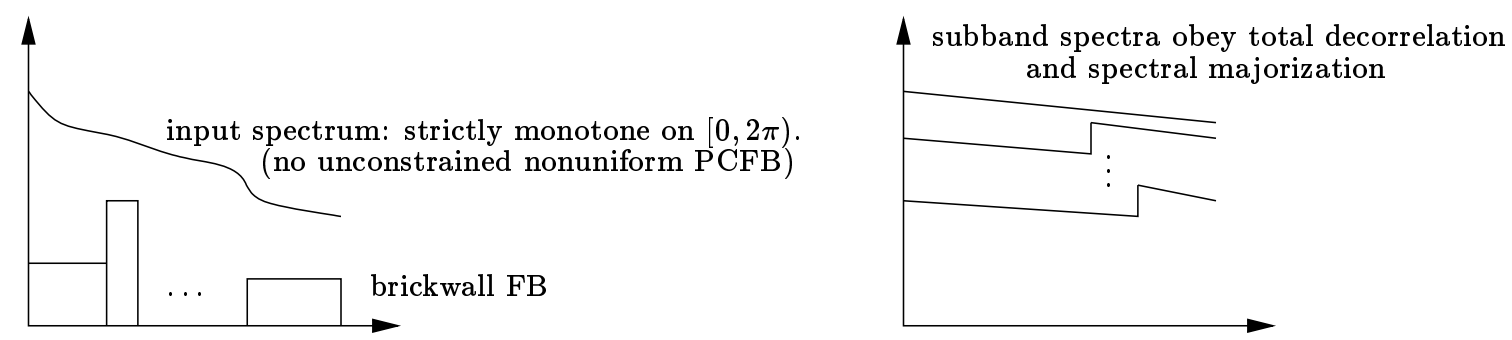

Fig. 4. Total decorrelation and spectral majorization do not imply nonuniform PCFB's.

\subsection{Proof of Theorem 1}

Without loss of generality, let $n_{0} \geq n_{1} \geq \ldots \geq n_{M-1}$. We prove the theorem for $a=0$ assuming that $S\left(e^{j \omega}\right)$ is strictly decreasing and that $n_{0} \neq n_{1}$ (i.e. $n_{0}>n_{1}$ ). The proof will show that this does not lose generality. The basic idea of the proof is as follows: Let $\mathcal{E}^{u}$ be the class of equivalent uniform FB's derived from FB's in $\mathcal{N}^{u}$. 
1. We apply the sequential compaction algorithm described in ${ }^{5}$ to the uniform FB class $\mathcal{E}^{u}$, and show that this results uniquely in the FB denoted by $F B_{A}$ in Fig. 5 (more precisely, in the equivalent uniform $\mathrm{FB}$ derived from $F B_{A}$ ). From ${ }^{5}$ this means that if the class $\mathcal{N}^{u}$ has a PCFB for the psd $S\left(e^{j \omega}\right)$, the PCFB must be $F B_{A}$.

2. We then show that $F B_{A}$ is not the PCFB by proving that its equivalent uniform subband variance vector does not majorize the variance vector of the distinct $\mathrm{FB}$ in $\mathcal{N}^{u}$ denoted by $F B_{B}$ in Fig. 5. This means that there is no PCFB.

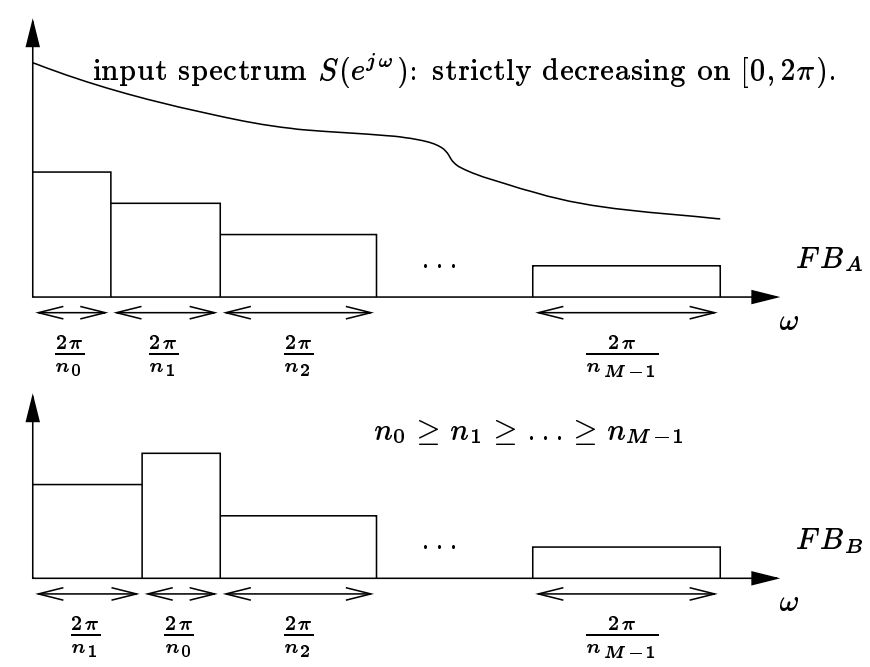

Fig. 5. Brickwall FB's used in proving Theorem 1.

Proof of Step 1: The sequential algorithm consists of maximizing the largest variance in the appropriate variance vector, then searching among all variance vectors having this maximum possible largest variance in order to maximize the second-largest variance, and so on. The entries of the equivalent uniform variance vector (8) are the nonuniform subband variances repeated appropriately many times. Hence running the algorithm on $\mathcal{E}^{u}$ is equivalent to sequentially maximizing the nonuniform FB subband variances. The ideal compaction $(M)$ filter ${ }^{12}$ produces the largest possible variance in a subband with decimator $M$, in any orthonormal FB. For a spectrum that is strictly decreasing on $[0,2 \pi)$ the compaction $(M)$ filter is unique, supported on $[0,2 \pi / M]$, and has output variance that is strictly increasing in $M$. As $n_{0}$ is the largest decimator in the FB (i.e. the largest possible $M$ ), this proves that among all FB's in $\mathcal{N}^{u}, F B_{A}$ maximizes the largest subband variance. (This maximum is the subband variance produced by the filter in $F B_{A}$ corresponding to decimator $n_{0}$.) Now all FB's are orthonormal, and so their analysis filters $H_{i}(z)$ satisfy $\left[H_{i}\left(e^{j \omega}\right) H_{j}^{*}\left(e^{j \omega}\right)\right] \downarrow_{\operatorname{gcd}\left(n_{i}, n_{j}\right)}=0$ for $i \neq j$. Hence, if an analysis filter in the $\mathrm{FB}$, corresponding to decimator $M$, has an aliasfree $(M)$ support, then this support does not overlap with the supports of any of the other analysis filters. Thus we can repeat the same argument for the second-largest variance, and so on. This shows that $F B_{A}$ is the unique output of the sequential compaction algorithm.

Proof of Step 2: If a filter in an orthonormal FB is ideal, supported on $[c, d]$ and has constant magnitude on its support (like all the filters in $F B_{A}, F B_{B}$ ), then the corresponding subband variance is given by direct calculation as

$$
\frac{f(d)-f(c)}{d-c}, \text { where } f(\omega)=\int_{0}^{\omega} S\left(e^{j \omega^{\prime}}\right) d \omega^{\prime} .
$$

The calculation uses the fact that the support length $d-c=2 \pi / n_{i}$ where $n_{i}$ is the corresponding channel decimator, and that the constant passband magnitude of the filter is $\sqrt{n_{i}}$ (both these are due to orthonormality). Notice that the variance expression (11) is the slope of the chord on the graph of $y=f(\omega)$, connecting the end points with abscissae $c, d$. In our case, $f$ is strictly concave on $[0,2 \pi)$, as its derivative is $S\left(e^{j \omega}\right)$ which is strictly decreasing. For chords of concave functions, increasing either or both of the abscissae of their endpoints can never cause the slope to increase. As a result, for both the brickwall FB's $F B_{A}$ and $F B_{B}$, the subband variance is nonincreasing as the 


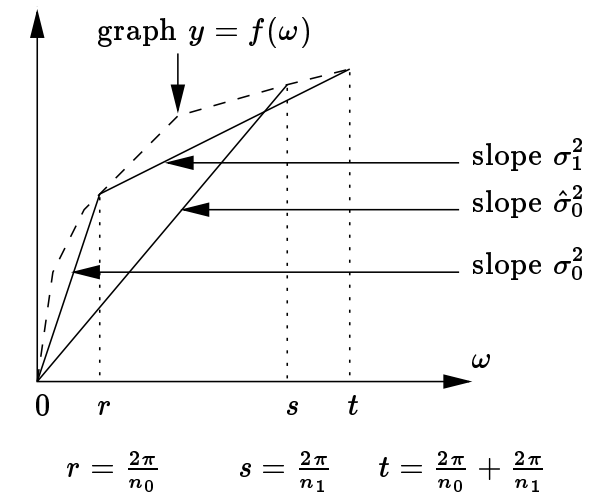

Fig. 6. Subband variances as chord slopes.

corresponding filter band-edges increase from 0 to $2 \pi$. Thus the largest and next-largest subband variances of $F B_{A}$ are $\sigma_{0}^{2}$ and $\sigma_{1}^{2}$, corresponding to decimators $n_{0}$ and $n_{1}$ respectively; while the largest subband variance of $F B_{B}$ is $\hat{\sigma}_{0}^{2}$ which corresponds to decimator $n_{1}$. All these variances are identifiable as slopes of chords on the graph $y=f(\omega)$ as in Fig. 6. Now we define $L=\operatorname{lcm}\left\{n_{i}\right\}$ and $p_{i}=L / n_{i}$, and let $\mathbf{v}_{A}, \mathbf{v}_{B}$ be the equivalent uniform subband variance vectors of $F B_{A}, F B_{B}$ respectively. We use the relation (8) between the subband variances of a nonuniform FB and its equivalent uniform FB. This shows that the $p_{1}$ largest variances in $\mathbf{v}_{B}$ have value $\hat{\sigma}_{0}^{2}$. On the other hand, of the $p_{1}$ largest variances in $\mathbf{v}_{A}$, there are $p_{0}$ variances of value $\sigma_{0}^{2}$ and $p_{1}-p_{0}$ variances of value $\sigma_{1}^{2}$. (Recall that $n_{0}>n_{1}$, so $p_{1}-p_{0}>0$.) Now referring to the definition (7) of majorization, we will have proved that the $\mathbf{v}_{A}$ does not majorize $\mathbf{v}_{B}$ if we show that

$$
p_{0} \sigma_{0}^{2}+\left(p_{1}-p_{0}\right) \sigma_{1}^{2}<p_{1} \hat{\sigma}_{0}^{2}
$$

To do this, we substitute the chord slopes from Fig. 6 for the variances. Using $p_{k}=L / n_{k}$ and deleting a factor of $L /(2 \pi)$, we see that proving the above equation reduces to proving that

$$
\frac{n_{1}}{n_{0}} f(r)+\left(1-\frac{n_{1}}{n_{0}}\right) f(t)<f(s)
$$

(where $r, s, t$ are as in Fig. 6). This is true by strict concavity of $f$, since $0<\frac{n_{1}}{n_{0}}<1$ and $s=\frac{n_{1}}{n_{0}} r+\left(1-\frac{n_{1}}{n_{0}}\right) t$.

Generalizing the proof. If the input spectrum is strictly increasing rather than decreasing on $[0,2 \pi)$ then very similar arguments hold $(f(\omega)$ is now convex). Alternatively we may observe that reflecting the frequency band $\omega \in[0,2 \pi)$ about $\omega=\pi$ gives a decreasing spectrum, and so a separate proof is really unnecessary. Similar comments hold if the interval of strict monotonicity is $[a, a+2 \pi)$ for some $a \neq 0$. If after arranging the decimators as $n_{0} \geq n_{1} \geq \ldots \geq n_{M-1}$ it happens that $n_{0}=n_{1}$, then we find the smallest $i$ for which $n_{i} \neq n_{i+1}$, and have $n_{i}, n_{i+1}$ play the roles played by $n_{0}, n_{1}$ respectively in the above proof. (There is such an $i$ because the FB's are not uniform.)

\section{CONCLUSION}

We have presented two different definitions of nonuniform principal component filter banks, generalizing the uniform ones. We have studied their optimality, showing that they minimize many concave objectives just like the uniform ones, though the form of the objective here must be somewhat more restricted in order to ensure PCFB optimality. We have shown that there are no nonuniform PCFB's for the unconstrained nonuniform FB classes if the input spectrum is strictly monotone (over an interval of length $2 \pi$ ). From this result we conclude that nonuniform PCFB existence is much more delicate than uniform PCFB existence, as it can be destroyed by small perturbations of the input spectra.

\section{REFERENCES}

1. S.Akkarakaran and P.P.Vaidyanathan, "The Role of Principal Component Filter Banks in Noise Reduction," in Proc. SPIE, Denver, CO, July 1999.

2. Sony Akkarakaran and P.P.Vaidyanathan, "Filter Bank Optimization with Convex Objectives, and the Optimality of Principal Component Forms," under review for IEEE Trans. Signal Processing. 
3. S.Akkarakaran and P.P.Vaidyanathan, "Are Nonuniform principal component filter banks optimal?," in Proc. EUSIPCO, Tampere, Finland, September 2000.

4. S.Akkarakaran and P.P.Vaidyanathan, "New results and open problems on nonuniform filter banks," in Proc. IEEE ICASSP, Phoenix, AZ, Mar. 1999.

5. Sony Akkarakaran and P.P.Vaidyanathan, "The best basis problem, compaction problem and PCFB design problems," in Proc. IEEE ISCAS, Orlando, FL, June 1999.

6. I.Djokovic and P.P.Vaidyanathan, "Results on Biorthogonal Filter Banks," Appl. Comput. Harmonic Analysis, vol. 1, pp. 329-343, 1994

7. R.A.Horn and C.R.Johnson, Matrix Analysis. Cambridge University Press, 1985.

8. A.Kirac and P.P.Vaidyanathan, "Optimal Nonuniform Orthonormal Filter Banks for Subband Coding and Signal Representation," in Proc. ICIP, Chicago, 1998.

9. J.Kovacevic and M.Vetterli, "Perfect Reconstruction Filter Bankswith Rational Sampling Factors," IEEE Trans. SP, vol. 41, pp. 2047-2066, June 1993.

10. M.K.Tsatsanis and G.B.Giannakis, "Principal Component Filter Banks for Optimal Multiresolution Analysis," IEEE Trans. Signal Processing, vol. 43, no. 8, pp. 1766-1777, Aug. 1995.

11. M.Unser, "An extension of the KLT for wavelets and perfect reconstruction filter banks," in Proc. SPIE no. 2034, Wavelet Appl. Signal Image Processing, San Diego, CA, 1993, pp.45-56.

12. P.P.Vaidyanathan, "Theory of Optimal Orthonormal Subband Coders," IEEE Trans. Signal Processing, vol. 46 , no. 6, pp. 1528-1543, June 1998.

13. P.P.Vaidyanathan, Yuan-Pei Lin, Sony Akkarakaran, and See-May Phoong, "Optimality of principal component filter banks for discrete multitone communication systems," in Proc. IEEE ISCAS, Geneva, May 2000.

14. P.P.Vaidyanathan, "Review of recent results on optimal orthonormal subband coders," in Proc. SPIE, San Diego, July 1997. 